\title{
ON COMPLEX OSCILLATION AND A PROBLEM OF OZAWA
}

\author{
By J. K. LANGLEY
}

\begin{abstract}
It is shown that if $Q(z)$ is a non-constant polynomial, then all non-trivial solutions of

$$
y^{\prime \prime}+\left(e^{z}+Q(z)\right) y=0
$$

have zeros with infinite exponent of convergence. Similar methods are used to settle a problem of M. Ozawa: if $P(z)$ is a non-constant polynomial, all non-trivial solutions of have infinite order.

$$
y^{\prime \prime}+e^{-z} y^{\prime}+P(z) y=0
$$
\end{abstract}

\section{Introduction.}

We are concerned with the order of growth of solutions of the differential equation

$$
y^{\prime \prime}+A(z) y^{\prime}+B(z) y=0
$$

where $A(z)$ and $B(z)$ are entire functions of finite order, not both polynomials. Denoting by $\sigma(g)$ the order of an entire function $g$, we note that if $\sigma(B)>\sigma(A)$ then it follows at once from the lemma of the logarithmic derivative (see [11]) that all non-trivial solutions of (1.1) are entire functions of infinite order. However, if $\sigma(B)<\sigma(A)$ it may be difficult to determine whether (1.1) can have non-trivial solutions of finite order, although since a finite order solution cannot grow large when $A(z)$ is close to its maximum modulus in this case, certain equations (for example if $A(z)=\sin \left(z^{n}\right)$ ) are easily dealt with.

Ozawa [13] considered the equation

$$
y^{\prime \prime}+e^{-z} y^{\prime}+P(z) y=0
$$

where $P(z)$ is a polynomial. A result of Frei [7] states that if $P$ is a constant, then (1.2) has a solution of finite order if and only if $P=-n^{2}$, where $n$ is an integer. (See also [3] for a result on the oscillation of solutions of (1.2) when $P$ is a constant). The case where $P$ is non-constant is more difficult to resolve and results of Ozawa [13], Amemiya and Ozawa [1], and Gundersen [9] may be summarised as follows:

Received May 1, 1986 
THEOREM A. Let $P(z)=a_{n} z^{n}+a_{p} z^{p}+\cdots+a_{0}$ be a non-constant polynomial. Then all non-trivial solutions of

$$
y^{\prime \prime}+e^{-z} y^{\prime}+P(z) y=0
$$

have infinite order if $P(z)$ satısfies any of the following:

(a) $n$ is odd;

(b) $n$ is even, and $n \geqq 2 p+3$;

(c) $n=2$, and $a_{1}=a_{0}=0$;

(d) $n$ is even, and $a_{n}(-1)^{n / 2}$ is not real and negatıve.

Now the problem of the growth of solutions of (1.1) is related to that of the oscillation of solutions of

$$
y^{\prime \prime}+A(z) y=0
$$

where $A(z)$ is a transcendental entire function of finite order. Denoting by $\lambda(g)$ the exponent of convergence of the zeros of $g$, it is shown in [2] that the product $E(z)$ of two linearly independent solutions $f_{1}$ and $f_{2}$ of (1.3) itself satisfies

$$
4 A=\left(E^{\prime} / E\right)^{2}-2\left(E^{\prime \prime} / E\right)-\left(1 / E^{2}\right)
$$

after a normalisation which makes tne Wronskian of $f_{1}$ and $f_{2}$ equal to 1 . Now (1.4) implies that if $\lambda(E)$ is finite then so is $\sigma(E)$ and moreover $E$ is small where $A$ is large so that if, for example, $\sigma(A)<\frac{1}{2}$ then $\lambda(E)$ must be infinite (see [2]). It is conjectured that if $\lambda(E)$ is finite then $\sigma(A)$ must be a positive integer. On the other hand examples of integer order do exist-there are pairs of polynomials $P$ and $Q$ (see [5]) whose degrees $d_{P}, d_{Q}$ satisfy

such that the equation

$$
d_{Q}+2=2 d_{P}
$$

$$
y^{\prime \prime}+\left(e^{P}+Q\right) y=0
$$

has two linearly independent non-vanishing solutions. These examples make the following result sharp [5]:

THEOREM B. Let $A(z)$ be a transcendental entire function of finite order $\rho$ with the following property: there exists a set $H \subseteq \boldsymbol{R}$, of measure zero, such that for each real $\theta$ not in $H$, either

or

(i) $r^{-N}\left|A\left(r e^{i \theta}\right)\right| \rightarrow \infty$ as $r \rightarrow \infty$, for each $N>0$,

(ii) $\int_{0}^{\infty} r\left|A\left(r e^{i \theta}\right)\right| d r<\infty$,

or

(iii) there exists $n \geqq 0$, possibly depending on $\theta$, such that $(n+2)<2 \rho$ and

$$
A\left(r e^{i \theta}\right)=O\left(r^{n}\right) \quad \text { as } \quad r \rightarrow \infty .
$$


Then if $f_{1}, f_{2}$ are linearly independent solutions of

$$
y^{\prime \prime}+A(z) y=0
$$

we have $\max \left\{\lambda\left(f_{1}\right), \lambda\left(f_{2}\right)\right\}=\infty$.

Now if (1.4) has a solution $E$ of finite order, which must be the product of two solutions of (1.3) (see [4]), then $E$ can only grow large where the growth of $A(z)$ is bounded by some power of $|z|$. In view of this fact, Theorem $\mathrm{B}$ and the examples preceding it, it is of interest to consider equations of the form (1.5), and the following was proved in [5]:

THEOREM C. Let $K \in C$ and suppose that

$$
f^{\prime \prime}+\left(e^{z}-K\right) f=0
$$

has a non-trivial solution with $\lambda(f)<\infty$.

\section{Then}

$$
K=q^{2} / 16
$$

where $q$ is an odd integer. Conversely if $K$ satisfies (1.7) with $q$ odd, then (1.6) has two linearly independent solutions $f_{1}, f_{2}$ with $\lambda\left(f_{1} f_{2}\right) \leqq 1$.

We remark that this result gives examples where $\sigma(E)$ is finite, but $E$ has zeros, although it seems worth noting that $\sigma(E)=\sigma(A)$ and the author is unaware of any examples of solutions of (1.4) for which $A$ is transcendental and

$$
\boldsymbol{\sigma}(A)<\boldsymbol{\sigma}(E)<\infty \text {. }
$$

In the case where $P$ is linear and $Q$ is non-constant we are able to prove the following:

THEOREM 1. Let $Q(z)$ be a non-constant polynomial, and let $\alpha \in C$. Then every non-trivial solution $f$ of

satisfies $\lambda(f)=\infty$.

$$
y^{\prime \prime}+\left(e^{z+\alpha}+Q(z)\right) y=0
$$

Now it turns out that (1.8) can only have a solution $f$ with $\lambda(f)<\infty$ if the equation

$$
y^{\prime \prime}+\left(2 c e^{z / 2}-\frac{1}{2}\right) y^{\prime}+\left(Q(z)+\frac{1}{16}\right) y=0,
$$

where $c \in C \backslash\{0\}$, has a solution of finite order (see $\S 3$ for a proof of this fact) and of course this equation is very close to (1.2). By similar methods to those of Theorem 1 we are able to settle Ozawa's problem:

THEOREM 2. Let $Q(z)$ be a non-constant polynomial. Then all non-trivial solutions of 


$$
y^{\prime \prime}+A e^{-z} y^{\prime}+Q(z) y=0
$$

have infinite order, for any $A \in C \backslash\{0\}$.

The author would like to acknowledge very valuable conversations with S. Bank.

\section{Notation and Preliminary Lemmas.}

We define an $R$-set to be a countable set of discs in the plane the sum of whose radii is finite, and remark, following Hayman [10], that the set of $\theta$ for which the ray $r e^{i \theta}$ meets infinitely many discs of a given $R$-set has measure zero.

A key role is played by the following lemma :

LEMMA 1. Let $S$ be the strip

$$
z=x+i y, \quad x \geqq x_{0}, \quad|y| \leqq 4 .
$$

Suppose that in $S$

$$
Q(z)=a_{n} z^{n}+O\left(|z|^{n-2}\right)
$$

where $n$ is a positive integer and $a_{n}>0$. Then there exists a path $\Gamma$ tending to infinity in $S$ such that all solutions of

tend to zero on $\Gamma$.

$$
y^{\prime \prime}+Q y=0
$$

Proof. We set $N=(n+2) / 2$ and

$$
Z=\int_{a}^{z} Q(t)^{1 / 2} d t
$$

for some large $a$ in $S$. Then $Z$ satisfies

$$
\begin{aligned}
Z & =\int_{a}^{z} k_{1} t^{n / 2}\left(1+O\left(t^{-2}\right)\right) d t \\
& =k_{2} z^{N}+o\left(|z|^{N-1}\right)
\end{aligned}
$$

in the smaller strip $S_{1}$ given by

$$
x \geqq x_{1}, \quad|y| \leqq 2 .
$$

Here $k_{1}, k_{2}, \cdots$ denote positive constants.

We assert that $Z$ is univalent in the strip $S_{2}$ given by

$$
x \geqq x_{2}, \quad|y| \leqq 1
$$

for some large $x_{2}$. For suppose that $z$ and $z_{1}$ are in $S_{2}$. Then since 


$$
z^{N}-z_{1}^{N}=\left(z^{N}+z_{1}^{N}\right)^{-1}\left(z-z_{1}\right) z_{1}^{2 N-1}\left(1+\cdots+\left(\frac{z}{z_{1}}\right)^{2 N-1}\right)
$$

we have

$$
\left|z^{N}-z_{1}^{N}\right| \geqq k_{3}\left|z-z_{1}\right| \max \left\{|z|^{N-1},\left|z_{1}\right|^{N-1}\right\}
$$

using the fact that $\operatorname{Re}\left(\left(z / z_{1}\right)^{j}\right)>0$ for $\jmath=1, \cdots, 2 N-1$ if $x_{2}$ is large enough. So (2.1) and (2.2) imply that if $z_{1}$ and $z_{2}$ are large with $Z\left(z_{1}\right)=Z\left(z_{2}\right)$, then $\left|z_{1}-z_{2}\right|$ $<1 / 2$. But then applying Rouché's theorem on the circle $\left|z-z_{1}\right|=1 / 2$, and using (2.1) and (2.2) we see that $Z(z)-Z\left(z_{1}\right)$ has but one zero in $\left|z-z_{1}\right|<1 / 2$.

We assert now that if $X$ is large and positive, $Z(z)$ takes the value $X$ at some point in $S_{2}$. This follows again from Rouché's theorem. Set $x_{3}=X^{1 / N}$. Then on the circle $\left|z-x_{3}\right|=1 / 2$, using (2.2), we have

$$
Z(z)-X=(1+o(1))\left(z^{N}-x_{3}^{N}\right) .
$$

We now choose $\Gamma$ to be the preimage in $S_{2}$ of the half line $\mathcal{L}$ given by

$$
Z=X, \quad X \geqq X_{0},
$$

with $X_{0}$ large. We make the standard transformation

$$
y(z)=Q(z)^{-(1 / 4)} U(Z)
$$

so that $U(Z)$ satisfies

$$
\frac{d^{2} U}{d Z^{2}}+(1-F(Z)) U=0
$$

where

$$
F(Z)=\frac{1}{4} \frac{Q^{\prime \prime}(z)}{Q(z)^{2}}-\frac{5}{16} \frac{Q^{\prime}(z)^{2}}{Q(z)^{3}} .
$$

Hence, for large $z$ in $S_{2}$ and $Z=Z(z)$,

so that writing

$$
\begin{aligned}
F(Z) & =O\left(|z|^{-(n+2)}\right) \\
& =O\left(|Z|^{-2}\right)
\end{aligned}
$$

$$
U(Z)=U_{1}(Z)+\int_{X_{0}}^{Z} \sin (Z-t) F(t) U(t) d t
$$

where $U_{1}$ satisfies

$$
U_{1}^{\prime \prime}+U_{1}=0
$$

we obtain

$$
|U| \leqq k_{4}+k_{5} \int_{X_{0}}^{Z}|F(t) U(t)| d t
$$

for $Z$ on $\mathcal{L}$ so that $U$ is bounded on $\mathcal{L}$ by Gronwall's lemma ([6], p 35). By (2.3) we see that $y(z) \rightarrow 0$ on $\Gamma$. 
We require the growth estimate below, deduced in [5] from Herold's comparison theorem [12]:

Lemma A. Suppose that $A(z)$ is analytic in a sector containing the ray $\mathcal{L}$ : $r e^{i \theta}$ and that as $r \rightarrow \infty$,

$$
A\left(r e^{i \theta}\right)=O\left(r^{n}\right)
$$

for some $n \geqq 0$. Then all solutions of

satısfy

$$
y^{\prime \prime}+A(z) y=0
$$

$$
\log ^{+}\left|y\left(r e^{i \theta}\right)\right|=O\left(r^{(n+2) / 2}\right)
$$

on $\mathcal{L}$.

\section{Proof of Theorem 1 .}

We assume that (1.8) has a solution $y(z)$ with $\lambda(y)<\infty$. By a translation we may assume that

$$
Q(z)=a_{n} z^{n}+a_{n-2} z^{n-2}+\cdots
$$

if $n \geqq 2$ or

$$
Q(z)=a_{1} z
$$

if $n=1$. We define the critical rays for $Q$ as those rays $r e^{i \theta}$ for which

$$
\operatorname{Arg} a_{n}+(n+2) \theta=0(\bmod 2 \pi)
$$

and remark that the substitution $z=x e^{i \theta}$ transforms the equation (1.8) into

$$
\frac{d^{2} y}{d x^{2}}+\left(e^{2 i \theta} e^{\alpha+x e^{i \theta}}+Q_{1}(x)\right) y=0,
$$

where $Q_{1}(x)=\alpha_{1} x^{n}+O\left(x^{n-2}\right)$ and $\alpha_{1}>0$. By Lemma 1, for any critical $\theta$ lying in $\left(\frac{\pi}{2}, \frac{3 \pi}{2}\right)$ there exists a path $\Gamma_{\theta}$ tending to infinity, such that arg $z \rightarrow \theta$ on $\Gamma_{\theta}$ while $y(z) \rightarrow 0$ there.

$A$ representation for $y(z)$.

Since $\lambda(y)<\infty$ we may write $y=\Pi e^{h}$ where $\sigma(\Pi)$ is finite, so that

$$
\frac{\Pi^{\prime \prime}}{\Pi}+\frac{2 \Pi^{\prime}}{\Pi} h^{\prime}+h^{\prime \prime}+h^{\prime 2}+e^{z+\alpha}+Q=0 \text {. }
$$

We differentiate (3.3) and subtract (3.3) from the differentiated equation, and find that

$$
h^{\prime}\left(2 h^{\prime \prime}-h^{\prime}\right)=P\left(h^{\prime}\right)
$$


where $P\left(h^{\prime}\right)$ is a differential polynomial in $h^{\prime}$, linear in $h^{\prime}, h^{\prime \prime}, \cdots$, and with coefficients which are differential polynomials in $\Pi^{\prime} / \Pi$. Clunie's Lemma applies ([11], p 68) and we deduce that

$$
2 h^{\prime \prime}-h^{\prime}=P_{1}(z)
$$

with $P_{1}$ a polynomial, so that

$$
h^{\prime}=c e^{z / 2}+P_{2}(z),
$$

where $c$ is a constant and $P_{2}$ is a polynomial. We then set

$$
y=W \exp \left(2 c e^{z / 2}-\frac{z}{4}\right)
$$

and substitute in the equation (1.8). (This device is due to S. Bank.) We remark that by (3.4) and (3.5), $W$ has finite order since $\Pi$ has.

Now $W$ satisfies

$$
W^{\prime \prime}+\left(2 c e^{z / 2}-\frac{1}{2}\right) W^{\prime}+\left(c^{2} e^{z}+e^{z+\alpha}+Q(z)+\frac{1}{16}\right) W=0 .
$$

Setting $V=W^{\prime} / W$ we have

$$
V^{\prime}+V^{2}+\left(2 c e^{z / 2}-\frac{1}{2}\right) V+\left(c^{2} e^{z}+e^{z+\alpha}+Q(z)+\frac{1}{16}\right)=0 .
$$

Since

$$
\left|V^{\prime}\right|+|V|=O\left(|z|^{M}\right)
$$

outside an $R$-set $U$ we deduce from (3.6) that $c^{2}=-e^{\alpha}$. Moreover, if $\phi \in$ $\left(\frac{-\pi}{2}, \frac{\pi}{2}\right)$ is such that the ray $r e^{\imath \phi}$ meets only finitely many discs of $U$ we see that $V=o\left(|z|^{-2}\right)$ as $z$ tends to infinity on this ray and hence that $W$ tends to a finite, non-zero limit. Applying this reasoning to a set of $\phi$ outside a set of zero measure we deduce by the Phragmén-Lindelöf principle that with no loss of generality, if $\varepsilon$ is positive, then

$$
W\left(r e^{i \theta}\right) \longrightarrow 1
$$

as $r \rightarrow \infty$ with $|\theta|<\frac{\pi}{2}-\varepsilon$. We deduce also from (3.5) that $W \rightarrow 0$ along the paths $\Gamma_{\theta}$ determined by the critical rays in the sector $\frac{\pi}{2}<\theta<\frac{3 \pi}{2}$.

The order of $W$.

By Lemma A, $y(z)$ satisfies

$$
\log ^{+}\left|y\left(r e^{i \theta}\right)\right|=O\left(r^{(n+2) / 2}\right)
$$

as $r \rightarrow \infty$, for any $\theta$ in $\left[\frac{\pi}{2}, \frac{3 \pi}{2}\right]$. Thus $(3.5),(3.7),(3.8)$ and the fact that $\varepsilon$ is 
arbitrary imply that, by the Phragmén-Lindelöf principle,

$$
\sigma(W) \leqq \frac{n+2}{2} .
$$

Conclusion of the proof.

If $n=1$, at least one critical $\theta$ for $Q(z)$ must lie in $\left(\frac{\pi}{2}, \frac{3 \pi}{2}\right)$. But this implies the existence of an unbounded domain of angular measure at most $\frac{\pi}{3}+$ $\varepsilon$, bounded by a path on which $W(z) \rightarrow 0$ and a ray on which $W(z) \rightarrow 1$. But then the Phragmén-Lindelöf Theorem (see eg. [8], p 104) implies that $\sigma(W) \geqq 3$, since $\varepsilon$ is arbitrary, and this contradicts (3.9).

In the case $n \geqq 2$ we claim that $\theta=\frac{\pi}{2}$ is a critical ray for $Q(z)$. For otherwise there exists a critical $\theta$ for $Q$ in

$$
\frac{\pi}{2}<\theta<\frac{\pi}{2}+\frac{2 \pi}{n+2}
$$

and the same reasoning as above, with

$$
\varepsilon=\frac{1}{2}\left(\frac{\pi}{2}+\frac{2 \pi}{n+2}-\theta\right)
$$

implies that $\sigma(W)>\frac{n+2}{2}$, contradicting (3.9). But now by Lemma $1, W(z) \rightarrow 0$ on a path $\Gamma_{\pi / 2}$ on which arg $z \rightarrow \frac{\pi}{2}$ and this combined with (3.7) provides a contradiction.

\section{Proof of Theorem 2.}

Since the proof is very similar to that of Theorem 1 we present only a sketch. Assuming that (1.9) has a solution $y(z)$ of finite order and that $Q(z)$ satisfies (3.1), or (3.2) if $Q$ is linear, we deduce as in the proof of Theorem 1 that, given any position $\varepsilon$, with no loss of generality

$$
y(z) \longrightarrow 1
$$

as $z=r e^{i \theta} \rightarrow \infty$ with $\frac{\pi}{2}+\varepsilon<\theta<\frac{3 \pi}{2}-\varepsilon$.

We now set

$$
y=u v
$$

where

$$
v=\exp \left(\frac{A}{2} e^{-z}\right)
$$

and $u$ satisfies 


$$
u^{\prime \prime}+\left(Q(z)-\frac{A^{2}}{4} e^{-2 z}+\frac{A}{2} e^{-z}\right) u=0 .
$$

Now, as $z \rightarrow \infty$ with $|\arg z|<\frac{\pi}{2}$, we see that $v(z) \rightarrow 1$, while on the strip

$$
z=x+i y, \quad|x| \leqq 4
$$

$|v(z)|$ is bounded above and below. Defining again the critical rays for $Q(z)$ as those rays $r e^{i \theta}$ for which

$$
\operatorname{Arg} a_{n}+(n+2) \theta=0 \quad(\bmod 2 \pi)
$$

we deduce from (4.2), (4.3), (4.4) and Lemma 1 that for any critical ray with $\theta$ lying in $\left(\frac{-\pi}{2}, \frac{\pi}{2}\right)$ there exists a path $\Gamma_{\theta}$ on which $u(z)$, and moreover $y(z)$, tend to zero, and on which arg $z \rightarrow \theta$, while the same is true if $n \geqq 2$ and $\theta= \pm$ $\frac{\pi}{2}$ is a critical ray.

As in the proof of Theorem 1 we obtain from Lemma A the estimate

$$
\sigma(y) \leqq \frac{n+2}{2},
$$

and proceed to a contradiction as follows. If $n=1$ some critical ray for $Q$ must lie in $\left(\frac{-\pi}{2}, \frac{\pi}{2}\right)$ and we find that $y(z) \rightarrow 1$ on a ray and $y(z) \rightarrow 0$ on a path which together bound a region of angular measure at most $\frac{\pi}{3}+\varepsilon$, implying that $\sigma(y)$ $>\frac{3}{2}$, which contradicts (4.5). On the other hand if $n \geqq 2$ the same reasoning implies that $\theta=\frac{\pi}{2}$ is a critical ray for $Q(z)$ and again we find that $y(z)$ tends to zero on a path $\Gamma_{\pi / 2}$. Since $y$ has finite order and $\varepsilon$ is arbitrary, this and (4.1) provide a contradiction.

\section{REFERENCES}

[1] I. Amemiya and M. Ozawa, Non-existence of finite order solutions of $w^{\prime \prime}+$ $e^{-z} w^{\prime}+Q(z) w=0$, Hokkaido Math. J., 10 (1981), Special Issue, 1-17.

[2] S. BANK AND I. LAINe, On the oscillation theory of $f^{\prime \prime}+A f=0$ where $A$ is entire, Trans. Amer. Math. Soc., 273, (1982), 351-363.

[3] S. BANK AND I. LAINE, Representation of solutions of periodic second order linear differential equations", J. Reine Angew. Math., 344 (1983), 1-21.

[4] S. BANK AND I. LAINE, On the zeros of meromorphic solutions of second order linear differential equations, Comment. Math. Helv., 58 (1983), 656-677.

[5] S. Bank, I. Laine AND J.K. LANGLey, On the frequency of zeros of solutions of second order linear differential equations, to appear, Resultate der Mathematik.

[6] R. Bellman, Stability theory of differential equations, McGraw-Hill, New York, 
1953.

[7] M. FREI, Über die subnormalen Lösungen der Differentialgleichung $w^{\prime \prime}+e^{-z} w^{\prime}$ + (const.) $w=0$, Comment. Math. Helv., 36 (1962), 1-8.

[8] W.H.J. Fuchs, Topics in the theory of functions of one complex variable, Van Nostrand Math. Studies, 12, 1967.

[9] G. Gundersen, On the question of whether $f^{\prime \prime}+e^{-z} f^{\prime}+B(z) f=0$ can admit a solution $f \equiv 0$ of finite order, Proc. R.S. Edinburgh, 102A (1986), 9-17.

[10] W.K. HAYMAN, Slowly growing integral and subharmonic functions, Comment. Math. Helv., 34 (1960), 75-84.

[11] W.K. Hayman, Meromorphic functions, Oxford at the Clarendon Press, 1964.

[12] H. Herold, Ein Vergleichssatz für komplexe linearer Differentialgleichungen, Math. Zeit., 126 (1972), 91-94.

[13] M. OzAwa, On a solution of $w^{\prime \prime}+e^{-z} w^{\prime}+(a z+b) w=0$, Kodai Math. J., 3 (1980), 295-309.

Department of Pure Mathematics,

Mathematical Institute,

North Haugh, St. Andrews,

Ky16 9SS, Scotland 